\title{
ABSOLUTE CONFIGURATION ASSIGNMENT IN RACEMIC trans-STILBENE OXIDE USING CHIRAL LIQUID CHROMATOGRAPHY WITH COMBINED CHIROPTICAL DETECTION AND TIME-DEPENDENT DENSITY FUNCTIONAL THEORY CALCULATIONS
}

\author{
JUAN P. CASTILLO-GONZÁLEZ, NATALIA I. GONZÁLEZ-PEÑA AND MARCELO A. MUÑOZ*
}

Instituto de Ciencias Quimicas, Facultad de Ciencias, Universidad Austral de Chile, Casilla 567, Valdivia, Chile.

\begin{abstract}
A chiral chromatographic analysis using HPLC coupled in series with circular dichroism and optical rotation detectors (HPLC-CD-OR) was applied to racemic trans-stilbene oxide, allowing the on-line measurement of the CD spectra and $[\alpha]_{670}$ values for both enantiomers in the sample. These measurements used the Gaussian peak model to estimate the concentration of each isomer in the CD and OR cells. Moreover, time-dependent density functional theory (TDDFT) calculations at the B3LYP/TZVP//B3LYP/VDZ(P) and B3LYP/aug-cc-pVDZ//B3LYP/VDZ(P) levels of theory for CD and OR, respectively, permitted the prediction of both chiroptical properties for each isomer, which in turn could be compared with the corresponding experimental data. In this case, the calculations were consistent in predicting the correct combination of experimental $\mathrm{CD}$ bands and $[\alpha]_{670}$ sign and therefore facilitated the absolute configuration (AC) assignment of the optical isomers. The $\mathrm{AC}$ proposed using this methodology was in agreement with independent $\mathrm{AC}$ assignments in the literature.
\end{abstract}

\section{INTRODUCTION}

The absolute configuration (AC) of chiral organic compounds is among the most difficult molecular properties to ascertain, and yet its importance is critical when studying biologically active substances of potential medicinal use. ${ }^{1}$ Conventional methods often involve complex procedures or require milligrams of high-purity samples, as is the case for chemical correlation, single-crystal X-ray diffraction, ${ }^{2}$ or electronic/vibrational circular dichroism spectroscopies. ${ }^{3}$ Methods based on chiroptical phenomena ${ }^{3}$ can clearly be more useful when coupled to chromatographic techniques, such as HPLC, enabling measurement of these properties in smaller and more complex samples and therefore extending their applicability.

Accordingly, HPLC techniques coupled with chiroptical detectors, mostly based in electronic circular dichroism (HPLC-CD) and optical rotation (HPLCOR), have been used in several applications, especially since commercial instrumentation has become available. ${ }^{4}$ Such applications include evaluation of novel chiral stationary phases ${ }^{5}$ determination of enantiomeric excess using non-chiral chromatography, ${ }^{6}$ and determination of the AC. ${ }^{7,8}$ HPLC-CD combined with theoretical calculations is preferred for AC assignments and has been applied to a wide range of compounds, especially natural products, in which the combined use of NMR and MS fully elucidated the structures of new substances without the need for conventional isolation. ${ }^{7}$ To our knowledge, no chromatographic measurements of specific optical rotation have been used in the stereochemical assignments of compounds of unknown AC to date. However, the sign of optical rotation measured in a wide wavelength range was recently used for this purpose. ${ }^{9}$

While advances in the calculation of chiroptical properties have furthered their applications in the AC determination of many systems, the potential risks in using a single chiroptical technique for these stereochemical assignments are significant, and the simultaneous use of at least two of these methods is strongly recommended to assign to a more reliable AC. . $^{10,13}$ An HPLC-CD-OR array permits the on-line measurement of two chiroptical properties simultaneously and, in conjunction with the corresponding quantum-chemical calculations, assigns the $\mathrm{AC}$ of the studied substance more confidently. This improvement in reliability is due to the potential agreement between both chiroptical properties, which can predict the same (or not) absolute configuration. The second measurement adds confidence to the initial assignment, particularly when $\mathrm{CD}$ measurements are obtained online using a dedicated detector with limited performance compared to a stand-alone CD spectrophotometer (i.e., shorter wavelength range and lower resolution).

While most of these applications assign the AC of single enantiomers, the use of this methodology along with chiral stationary phases (CSP) can determine the $\mathrm{AC}$ of chiral compounds even when part of a racemic or partially enriched mixture. Such capability is needed in many applications, including the non-asymmetric chemical synthesis of chiral compounds, in which the AC of racemic products and intermediaries could be assigned without the need of enantioselective isolation. Additionally, the complementary use of CD and OR detection will be useful when applied to libraries of racemic building blocks that, although commercially available, often lack information about their ACs.

We recently reported the use of this approach in the study of the chiroptical properties of a racemic flavanone that agreed with VCD observations and literature data. ${ }^{14}$

In this work, we further the use of chiral HPLC-CD-OR analysis and time-dependent density functional theory (TD-DFT) calculations for the AC assignment of racemic mixtures by applying it to racemic trans-stilbene oxide (1).

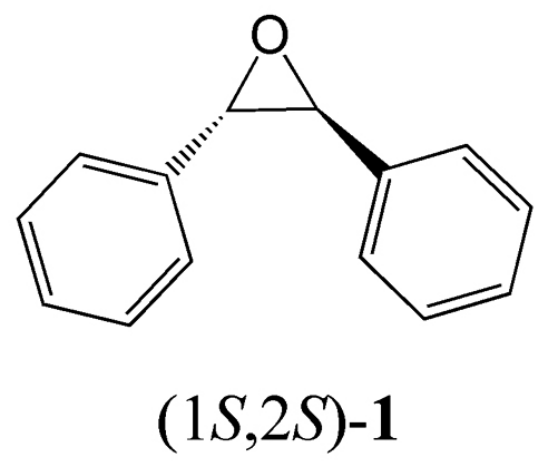

Fig. 1. Structure of trans-stilbene oxide (1). Only one enantiomer is shown.

\section{EXPERIMENTAL}

\subsection{Reagents and chemicals}

$( \pm)$-trans-Stilbene oxide (98\%) was acquired from Aldrich (Sigma-Aldrich Química Ltda., Chile). HPLC-grade solvents n-hexane and 2-propanol were acquired from Merck (Merck S.A., Chile). All chemicals and solvents were used directly without further purification.

2.2 HPLC instrumentation and analyses

Chromatographic analyses were performed on a Shimadzu HPLC chromatograph equipped with LC-20AT and LC-10ADvp pumps, a SIL10Avp autosampler, and a CTO-10ACvp column oven. Coupled in series to this array, a JASCO CD-2095plus UV-CD detector with a $220-420 \mathrm{~nm}$ range and equipped with a $25-\mathrm{mm}$ path length and $44-\mu \mathrm{L}$ internal volume flow cell was used for CD measurements. The latter was followed in-line by a PDRChemical LLR advanced laser polarimeter detector equipped with a $670-\mathrm{nm}$ diode laser and a $25-\mathrm{mm}$ path length $(18-\mu \mathrm{L}$ internal volume flow cell), which was used for OR measurements. UV, CD and OR signals from both chiroptical detectors were acquired with JASCO ChromPass software version 1.7.403.1.

Chiral separations were then performed on a ChiralPak AD-H column ( 250 $\mathrm{mm}, 4.5 \mathrm{~mm}, 5 \mu \mathrm{m}$, Daicel Inc.) with a mixture of n-hexane and 2-propanol 9:1 as the mobile phase keeping the chiral column at $25.0^{\circ} \mathrm{C}$ during the analyses.

UV and CD spectra were acquired simultaneously for both enantiomers in each racemic mixture using the stop-flow technique ${ }^{15}$ and the mobile phase as the baseline. Additionally, five consecutive measurements were made for each eluted peak, which substantially decreased the noise of the UV and CD 
spectra. Identical UV spectra and antipodic CD spectra were obtained for each enantiomeric pair, discarding the presence of bands produced by low transmission, particularly at shorter wavelengths. Solutions of $\mathbf{1}$ with appropriate concentrations (between 1 and $10 \mu \mathrm{g} / \mu \mathrm{L}$ ) were prepared by dissolving in the corresponding mobile phase in 5-mL volumetric flasks, and samples of these solutions were filtered and analysed using 1 - to $20-\mu \mathrm{L}$ injections.

$[\alpha]_{670}$ values were extracted by measuring the height and half-height width of each OR peak and then applying the Gaussian peak model. ${ }^{16}$ Likewise, the concentration of each stereoisomer in the flow cell during CD measurements was estimated using the same approach. This permitted the conversion of experimental $\mathrm{CD}$ intensity into molar $\mathrm{CD}$ units.

\subsection{Computational calculations}

Conformational searches were performed using the molecular mechanics force field (MMFF), as implemented in the Spartan'14 software (Wavefunction Inc, Irvine, CA 92612, USA). All conformations found in the first $10 \mathrm{kcal} /$ mol were submitted to geometry optimization and vibrational calculations at the B3LYP/VDZ(P) level of theory. To assess the influence of solvent in the conformational distribution and molecular geometry, the same procedure was performed separately using the conductor-like screening model (COSMO $)^{17}$ with a dielectric constant equivalent to that of the mobile phase. Using the free energy values obtained from these calculations on a Boltzmann distribution produced relative abundances for each conformation, and only those found to be relevant were considered for $\mathrm{CD}$ and $\mathrm{OR}$ calculations. Calculated $\mathrm{CD}$ spectra for each individual conformation were obtained from the rotational strengths of the first 10 single excited states calculated at the TD-DFT B3LYP/TZVP level of theory, which were then plotted as Gaussian bands with half-widths of $15 \mathrm{~nm}$ using the SpecDis software package. ${ }^{18}$ In addition, a global shift of the calculated spectra to shorter wavelengths was needed to compensate for the tendency of TD-DFT to underestimate excitation energies. ${ }^{19}$ This shift was determined visually using the UV and CD spectra. The average optical rotation tensor $\beta$ was calculated for each considered conformation at the B3LYP/augcc-pVDZ level of theory and then converted to $[\alpha]_{670}$ values as previously described. ${ }^{20}$ Finally, single weighted CD spectra and $[\alpha]_{670}$ values were obtained by considering the relative abundances of the corresponding Boltzmann distribution. Geometry optimizations and free energy calculations were carried out using the ORCA software package, ${ }^{21}$ while TD-DFT calculations were carried out using the NWChem software package. ${ }^{22}$ Although many different combinations of functional and basis sets have been used successfully for the calculation of these chiroptical properties, the popular B3LYP functional along with DGauss double- $\zeta$ plus polarization (geometries), DGauss triple- $\zeta$ plus polarization $(\mathrm{CD})$ and double- $\zeta$ augmented Dunning's correlation consistent (OR) basis sets are common choices in the literature. ${ }^{20,23}$ They offer a good balance between accuracy and computational cost and are readily available in many computational chemistry software packages.

\section{RESULTS AND DISCUSSION}

Compound $\mathbf{1}$ is a chiral pro-estrogen used to induce liver drug-metabolizing enzymes and, more recently, has been shown to induce the expression of genes involved in metabolism and transport in the mouse liver. ${ }^{24}$ It is also a standard compound for testing CSP under normal phase conditions and is frequently encountered in catalogues and test chromatograms for commercially available chiral HPLC columns. ${ }^{25}$ Accordingly, chromatographic resolution $(\mathrm{Rs}=10.6)$ was easily achieved using an amylose-derived CSP, and peak elution was monitored by UV, CD and OR detections. The first peak to elute (5.5 min) corresponded to $(+)-\mathbf{1}$, and the second (10.4 min) was (-)-1 (Fig. 2).

This also permitted the extraction of $[\alpha]_{670}$ values from the OR chromatographic peaks using the Gaussian peak approach (207.0 and -200.9 for the first and second eluted peaks, respectively), revealing a high optical activity characteristic of molecules bearing inherently dissymmetric chromophores ${ }^{26}$, even in the absence of formal conjugation. ${ }^{27}$ In a second run, the CD spectra of each isomer was measured in the $220-420 \mathrm{~nm}$ range using the stop-flow technique, which now produced a positive Cotton effect only for the first eluted peak and the corresponding antipodal response for the second (Fig. 3).

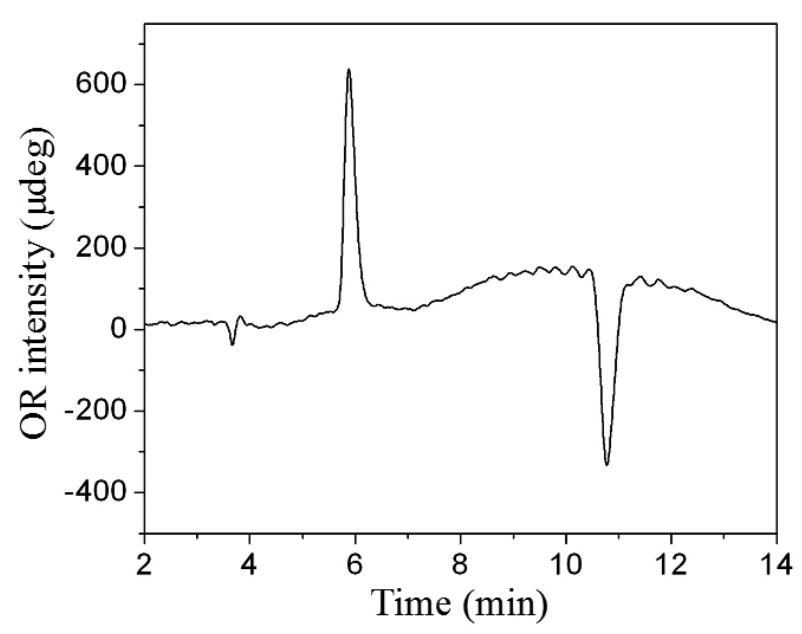

Fig. 2. OR chromatogram of the enantioselective separation of ( \pm -1: detection at $670 \mathrm{~nm}$.

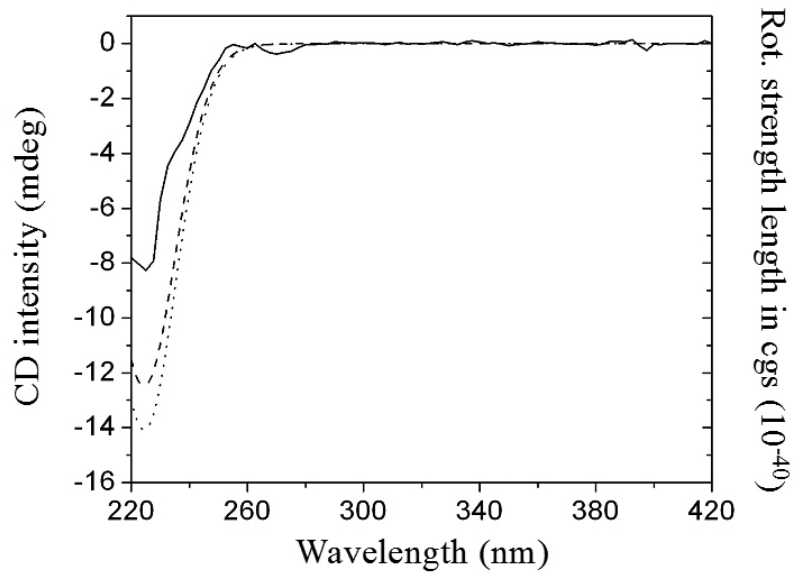

Fig. 3. Experimental (continuous), calculated in the gas phase (dashed) and calculated in solution (dotted) CD spectra of (-)-(1S,2S)-1 (flow cell concentration of $\left.1.5 \times 10^{-5} \mathrm{M}\right)$. The CD spectrum was calculated at the B3LYP/ $\mathrm{TZVP} / / \mathrm{B} 3 \mathrm{LYP} / \mathrm{VDZ}(\mathrm{P})$ level of theory and shifted $20 \mathrm{~nm}$ towards shorter wavelengths for better comparison with the experimental data.

On the other hand, a systematic conformational search showed only one low-energy conformation, in which the plane of each phenyl group is oriented almost parallel to the $\mathrm{C}-\mathrm{O}$ oxirane bond (O-C-Cipso-Corto dihedral angles of $-10.7^{\circ}$ ) and thus forming a $-57.5^{\circ}$ angle between both aromatic rings (Fig. 4).
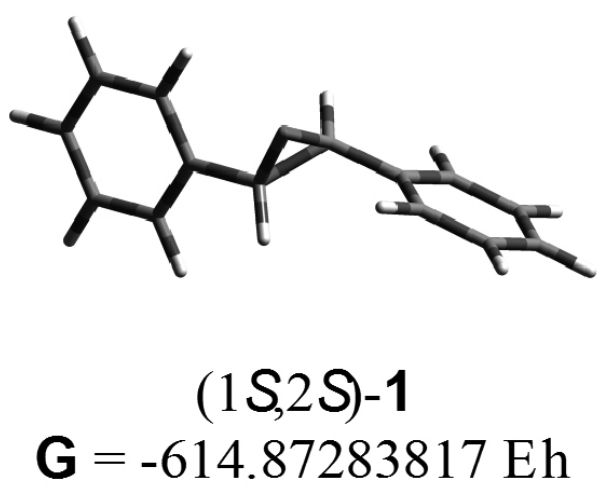

Fig. 4. Geometry optimized conformation and Gibbs free enthalpy $(\mathrm{G})$ of $(1 S, 2 S)-\mathbf{1}$ at the B3LYP/VDZ(P) level of theory. 
Consideration of solvent effects using a dielectric constant equivalent to that of the given mobile phase, led to a very similar conformation with small changes in these angles $\left(-12.6^{\circ}\right.$ and $-61.0^{\circ}$, respectively). Using both conformations, calculated CD spectra and $[\alpha]_{670}$ values for each enantiomer were obtained and compared with the corresponding experimental data. As shown in Fig. 3, the CD spectra calculated for the $(1 S, 2 S)$ isomer agreed with the experimental spectrum of (-)-1 (the second eluted peak). The calculated $[\alpha]_{670}$ value of -212.9 for the $(1 S, 2 S)$ isomer showed an excellent correlation with the measured value, both in sign and magnitude, and therefore confirmed the $\mathrm{CD}$ results by predicting this isomer to be levorotatory. Consideration of solvent effects did not lead to better agreement with experimental values in this case $(-184.3$ for the $(1 S, 2 S)$ isomer), but the differences observed between properties calculated in the gas and solution states are noteworthy. Very small differences between these low energy conformers produced noticeable changes in $\mathrm{CD}$ and $\mathrm{OR}$ intensities (although not in their signs), suggesting that solvent consideration might be of importance in more complex cases.

From these comparisons, the $\mathrm{AC}$ of $\mathbf{1}$ is $(+)-(1 R, 2 R)-\mathbf{1}$ and $(-)-(1 S, 2 S)-\mathbf{1}$, which is in agreement with previous studies based on chemical correlation and application of the exciton chirality rule. ${ }^{26,28}$

\section{CONCLUSIONS}

The use of chiral HPLC with CD and OR detection (HPLC-CD-OR), along with TD-DFT computational calculations, is well suited to assign the absolute configuration of both enantiomers in racemic trans-stilbene oxide (1). Excellent chromatographic resolution, high optical activity and a single lowenergy conformation were obtained for $\mathbf{1}$, and calculation and comparison of both chiroptical properties gave an AC of (+)-(1R,2R)-1 and (-)-(1S,2S)-1.

\section{ACKNOWLEDGEMENTS}

The authors acknowledge financial support from Universidad Austral de Chile (project DID-UACH S2013-02) and CONICYT-Chile (project FONDECYT 1141071).

\section{REFERENCES}

1 Q. Shen, L. Wang, H. Zhou, H. Jiang, L. Yu, Su Zeng, Acta Pharmacol. Sin. 34, 998-1006, (2013)

2 H.D. Flack, G. Bernardinelli, Chirality 20, 681-690, (2008)

3 G.H. Wagnière, On the interaction of light with molecules: pathways to the theoretical interpretation of chiroptical phenomena, in: N. Berova, P.L. Polavarapu, K. Nakanishi, R.W. Woody (Eds.), Comprehensive Chiroptical Spectroscopy Vol. 1, John Wiley \& Sons, New York, 2012, pp. 3-34.

4 L. Kott, W.B. Holzheuer, M.M. Wong, G.K. Webster, J. Pharm. Biomed. Anal. 43, 57-65, (2007)

5 X.-M. Chena, C. Yamamotob, Y. Okamoto, J. Chromatogr. A 1104, 62-68, (2006)

6 E. Bossu', V. Cotichini, G. Gostoli, A. Farina, J. Pharm. Biomed. Anal. 26, $837-848,(2001)$

7 G. Bringmann, T.A.M. Gulder, M. Reichert, T. Gulder, Chirality 20, 628642, (2008)

8 N. Vanthuyne, C. Roussel, Chiroptical detectors for the study of unusual phenomena in chiral chromatography, in: V. Schurig (Ed.), Differentiation of Enantiomers I, Topics in Current Chemistry Volume 340, Springer International Publishing, 2013, pp. 107-151.

9 K. Szwed, M. Górecki, J. Frelek, M. Asztemborska, Chromatographia 76, 1603-1611, (2013)

10 P.J. Stephens, J.J. Pan, F.J. Devlin, K. Krohn, T. Kurta'n, J. Org. Chem. 72 , 3521-3526, (2007)

11 P.J. Stephens, J.-J. Pan, F.J. Devlin, M. Urbanová, J. Hájíček, J. Org. Chem. 72, 2508-2524, (2007)

12 P.J. Stephens, F.J. Devlin, F. Gasparrini, A. Ciogli, D. Spinelli, B. Cosimelli, J. Org. Chem. 72, 4707-4715, (2007)

13 P.J. Stephens, D.M. McCann, F.J. Devlin, A.B. Smith III, J. Nat. Prod. 69, 1055-1064, (2006)

14 M.A. Muñoz, M.A. Bucio, P. Joseph-Nathan, Nat. Prod. Commun. 8, 1075-1078, (2013)

15 C. Bertucci, D. Tedesco, J. Chromatogr. A 1269, 69-81, (2012)

16 P.D. Rice, Y.Y. Shao, S.R. Erskine, T.G. Teague, D.R. Bobbitt, Talanta 36, 473-478, (1989)
17S. Sinnecker, A. Rajendran, A. Klamt, M. Diedenhofen, F. Neese, J. Phys. Chem. A. 110, 2235-2245, (2006)

18 T. Bruhn, A. Schaumlöffel, Y. Hemberger, G. Bringmann, SpecDis version 1.62, University of Wuerzburg, Germany, 2014. (http://www-organik. chemie.uni-wuerzburg.de/lehrstuehlearbeitskreise/bringmann/specdis/)

19 R. Bauernschmitt, R. Ahlrichs, Chem. Phys. Lett. 256, 454-464, (1996)

20 M. Srebro, N. Govind, W. A. de Jong, J. Autschbach, J. Phys. Chem. A 115, 10930-10949, (2011)

21 F. Neese, WIREs Comput. Mol. Sci. 2, 73-78, (2012)

22 M. Valiev, E.J. Bylaska, N. Govind, K. Kowalski, T.P. Straatsma, H.J.J. van Dam, D. Wang, J. Nieplocha, E. Apra, T.L. Windus, W.A. de Jong, Comput. Phys. Commun. 181, 1477-1489, (2010)

23 N. Berova, L.D. Bari, G. Pescitelli, Chem. Soc. Rev. 36, 914-931, (2007)

24 A.L. Slitt, N.J. Cherrington, M.Z. Dieter, L.M. Aleksunes, G.L. Scheffer, W. Huang, D.D. Moore, C.D. Klaassen, Mol. Pharmacol. 69, 1554-1563, (2006)

25 Lux Columns Complete Chiral Solutions Catalogue, Phenomenex, Torrance, CA, USA (2013).

26 G. Gottarelli, S. F. Mason, G. Torre, J. Chem. Soc. B, 1349-1353, (1970)

27 K. Mislow, Introduction to Stereochemistry, first ed., W. A. Benjamin, Inc., New York, 1966.

28 M. Imuta, H. Ziffer, J. Org. Chem. 44, 2505-2509, (1979) 